\title{
Alantolactone suppresses the metastatic phenotype and induces the apoptosis of glioblastoma cells by targeting LIMK kinase activity and activating the cofilin/G-actin signaling cascade
}

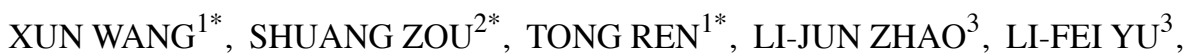 \\ XIANG-YU LI ${ }^{1}$, XIN YAN $^{4}$ and LI-JUN ZHANG ${ }^{3}$
}

\author{
${ }^{1}$ Department of Neurosurgery, The Third People's Hospital of Dalian, Non-Directly Affiliated Hospital of \\ Dalian Medical University, Dalian, Liaoning $116033 ;{ }^{2}$ Department of Neurology, Second Affiliated Hospital, \\ Zhejiang University School of Medicine, Hangzhou, Zhejiang 310009; \\ Departments of ${ }^{3}$ Ophthalmology and ${ }^{4}$ Medical Oncology, The Third People's Hospital of Dalian, \\ Non-Directly Affiliated Hospital of Dalian Medical University, Dalian, Liaoning 116033, P.R. China
}

Received December 3, 2020; Accepted February 5, 2021

DOI: $10.3892 /$ ijmm.2021.4901

\begin{abstract}
Glioblastoma (GBM) is the most common aggressive brain tumor and is associated with an extremely poor prognosis, as the current standard of care treatments have limited efficacy. Natural compounds have attracted increasing attention as potential anticancer drugs. Alantolactone (ATL) is a natural small molecule inhibitor, that has antitumor properties. In the present study, U87MG and U251 cells were treated ATL and changes in actin/G-actin/F-actin/cofilin pathway were detected in whole cells, in the cytoplasm and mitochondria by western blot analysis. Immunofluorescence and immunoprecipitation analysis identified changes in the expression levels of target proteins and interactions, respectively. A LIMK enzyme inhibitor was also applied to assess the effects of ATL on the migration and invasion of GBM cells. Flow cytometry was used to detect the levels of apoptosis of GBM cells. The expression of matrix metalloproteinase (MMP)-2/MMP-9, caspase-3/caspase-9/poly(ADP-ribose) polymerase (PARP)/cytochrome $c$, were determined by western blot analysis to assess the effects of targeting LIMK. The in vitro
\end{abstract}

Correspondence to: Professor Li-Jun Zhang, Department of Ophthalmology, The Third People's Hospital of Dalian, Non-Directly Affiliated Hospital of Dalian Medical University, 40 Qianshan Road, Ganjingzi, Dalian, Liaoning 116033, P.R. China

E-mail: zhanglijundl@126.com

Professor Xin Yan, Department of Medical Oncology, The Third People's Hospital of Dalian, Non-Directly Affiliated Hospital of Dalian Medical University, 40 Qianshan Road, Ganjingzi, Dalian, Liaoning 116033, P.R. China

E-mail: yanxindl@126.com

${ }^{*}$ Contributed equally

Key words: alantolactone, glioblastoma, LIMK, cofilin, migration and invasion, apoptosis findings were verified in vivo by characterizing changes in the expression of cofilin/LIMK in xenograft tumors in immunodeficient mice. It was found that ATL activated cofilin through the targeted inhibition of LIMK enzyme activity and it thus upregulated the ratio of G/F actin, and inhibited GBM cell migration and invasion. Conversely, the activation of cofilin and G-actin could be co-transferred to the mitochondria to initiate the mitochondrial-cytochrome $c$ pathway to induce apoptosis. On the whole, the findings of the present study further illustrate the molecular mechanisms through which ATL inhibits the metastatic phenotype of GBM cells and induces apoptosis. Given previous findings, it can be deduced that ATL can function through multiple pathways and has multiple targets in GBM models, highlighting its potential for use in clinical applications.

\section{Introduction}

Glioblastoma (GBM) is a devastating disease that has an extremely poor prognosis. The 5-year survival rate of patients with GBM is $<3 \%$ and the 5-year mortality rate ranks third amongst all malignant tumors (1-3). The high mortality rate is due to the invasive nature of GBM, as tumors can spread locally within the brain or disseminate to other sites within the body (4). There is a critical need for the better understanding of the molecular mechanisms that drive the invasive phenotype of GBM so that improved therapeutic approaches can be developed. Moreover, the identification of natural drugs with efficacy in the treatment of GBM have a high potential to lead to the development of novel therapies that may benefit a large number of patients.

Alantolactone (ATL; chemical structure shown in Fig. 1A) is a semi-terpene lactone compound that is isolated from the roots of Inula helenium. ATL has potent biological activities that include anti-inflammatory, antitumor, anti-parasitic and hepatoprotective properties (5). Previously, the authors demonstrated that ATL can pass through the blood-brain barrier to inhibit the growth and metastatic phenotype of GBM and 
also induce apoptosis (6). However, the underlying molecular mechanisms of these effects and the regulatory targets activated by ATL remain to be fully characterized.

Cell migration is a critical hallmark of tumor invasion and metastasis (7). A detailed investigation of the mechanisms of cell migration has revealed that cell polarity, the dynamic regulation of depolymerization, the polymerization of microfilaments and microtubules, and changes in signal transduction during migration are all related to the recombination of the actin cytoskeleton (8).

Actin is a protein that is divided into a polymer form of F-actin (fibrous) and a monomer form of G-actin (spherical). The formation of pseudopodia is dependent on the polymerization and depolymerization of actin. Cofilin is a binding protein of actin and represents a family of actin depolymerization factors that dominates the migration of cells. Cofilin induces the depolymerization of fibrous F-actin into spherical G-actin, leading to the remodeling of the cytoskeleton. This process affects cell migration and occurs during embryonic development, wound repair and tumor cell invasion and metastasis (9).

LIMK is a serine/threonine-protein kinase that includes 2 members, LIMK1 and LIMK2. These proteins catalyze the phosphorylation of cofilin as upstream molecules that regulate cofilin. LIMK inactivates cofilin by phosphorylating the serine residue at the 3 rd position of cofilin protein. It also mediates actin polymerization to form a pseudopodoid structure and can regulate cofilin activity through changes in the enzyme activity of LIMK to control the migration and invasion properties of tumor cells (10). Studies have found that LIMK is involved in the progression, migration and invasion of various malignant tumors, including ovarian, breast and colon cancer cells (11-13). It has also been demonstrated that the translocation of activated cofilin to the mitochondria is an early marker of apoptosis. Cofilin translocates to the outer membrane of the mitochondria and releases cytochrome $c$ into the cytoplasm to induce apoptosis (14)

Based on these previous findings, it was hypothesized that ATL may activate cofilin through the targeted inhibition of LIMK enzyme activity. This may inhibit cell migration and invasion and also induce apoptosis. In the present study, in vitro and in vivo experiments were performed to investigate the molecular mechanisms of ATL in modulating the migration, invasion and apoptosis of GBM cells. The data of the present study support the further development of ATL as a potential therapy for the treatment of GBM.

\section{Materials and methods}

Chemicals and antibodies. ATL ( $\geq 98.5 \%$ purity) was prepared by the laboratory at Dalian Medical University. Extraction and purification were performed via stepwise elution in a solvent system containing hexane:ethyl acetate:methanol:water at volumetric ratios of 5:3:1:2(v/v). The concentration of the ATL stock solution was $100 \mu \mathrm{mol} / \mathrm{l}$. The stock solution was dissolved in dimethyl sulfoxide (DMSO) and stored at $-20^{\circ} \mathrm{C}$ for further experimental use. The final concentration of DMSO was $<0.1 \%$ when ATL was applied to the cells. The LIM kinase Inhibitor I (LIMKi 3) was purchased from MedChem Express. Antibodies against cofilin (sc-376476), LIMK1 (sc-28370)/2 (sc-365414) and phospho-LIMK1/2 (Thr508/505) (sc-28409) were purchased from Santa Cruz Biotechnology, Inc. Antibodies against phospho-cofilin (Ser 3) (cat. no. 3311), cytochrome $c$ oxidase (Cox) IV (cat. no. 4844), slingshot homolog (SSH; cat. no. 13578), testis associated actin remodeling kinase (cat. no. 4655, TESK), GAPDH (cat. no. 2118), $\beta$-actin (cat. no. 4970), cleaved caspase-3 (cat. no. 9664)/9 (cat. no. 20750)/poly(ADP-ribose) polymerase (cat. no. 5625, PARP) were purchased from Cell Signaling Technology, Inc. Matrix metalloproteinase (MMP)-2 (cat. no. 66366-1-Ig)/9 (cat. no. 10375-2-AP) and cytochrome $c$ (cat. no. 10993-1-AP) antibodies were purchased from Proteintech Group, Inc. and actin (cat. no. A4700) antibody was from Sigma-Aldrich; Merck KGaA.

Cells and cell culture. The U87MG (cat. no. HTB-14) and U251 cell lines were obtained from the American Type Culture Collection (ATCC). All cells were maintained in DMEM supplemented with $10 \%$ fetal bovine serum (FBS) and maintained at $37^{\circ} \mathrm{C}$ in a humidified atmosphere containing $5 \% \mathrm{CO}_{2}$.

The U87MG cell line is a glioblastoma of unknown origin. The authenticity of the cell line was verified through the genomic short tandem repeat profile by Shanghai Biowing Biotechnology Co. Ltd. and the cell line were confirmed to be free of mycoplasma using a Mycoplasma Detection kit-Quick Test (Biotool, LLC).

Wound healing migration assay. The cells were allowed to grow to become fully confluent in 6-well plates. A line of cells was then scraped away in each well using a pipette tip after $6 \mathrm{~h}$ of serum starvation. The cells were then washed twice to remove detached cells. Fresh medium containing different drug treatments (DMSO; ATL, $10 \mu \mathrm{M}$; LIMKi 3, $5 \mu \mathrm{M}$; and ATL + LIMKi 3) were added to the scratched monolayers. Images were acquired using a Leica DM 14000B microscope following $24 \mathrm{~h}$ of incubation at $37^{\circ} \mathrm{C}$. The migrating cells were observed from 3 randomly chosen fields of view and quantified by manual counting. The percentage inhibition of migration was expressed as relative to the untreated cells (100\%).

Transwell invasion assay. Liquefied matrix glue was mixed with DMEM (1:3 ratio) and kept on ice. A total of $80 \mu \mathrm{l}$ of the mixture was applied to the cell membrane, and incubated at $37^{\circ} \mathrm{C}$ for $30 \mathrm{~min}$ to solidify the matrix glue. Cells were trypsinized and resuspended in culture medium without fetal bovine serum. Subsequently, $100 \mu 1$ of cell suspension (density $5 \times 10^{5}$ cells $/ \mathrm{ml}$ ) were added to the upper layer of the chamber, which were pre-coated with Matrigel (BD Biosciences) and $700 \mu 1$ of DMEM medium containing fetal bovine serum was added to the lower layer. The upper and lower layers of each group contained the same concentration of cells in each of the treatment groups. Following incubation for $24 \mathrm{~h}$ at $37^{\circ} \mathrm{C}$, cotton swabs were used to remove the residual matrix glue and cell suspension in the upper chamber. The chamber was then fixed in methanol for $15 \mathrm{~min}$. After removing the chamber and allowing the membrane to air dry, the chamber was stained with $1 \%$ crystal violet (Sigma-Aldrich; Merck KGaA) for $20 \mathrm{~min}$ at room temperature and washed 3 times with PBS. The invading cells were detected after staining under the membrane of the chamber were photographed under a Leica DM 14000B microscope (Leica Microsystems GmbH). 
Flow cytometry analysis. Cells were seeds in 6-cm culture dishes and treated with the different drugs (DMSO; ATL, $10 \mu \mathrm{M}$; LIMKi 3, $5 \mu \mathrm{M}$; and ATL + LIMKi 3) for $24 \mathrm{~h}$. The cells were then washed with PBS twice and trypsinized. The cells were collected by centrifugation $(400 \times \mathrm{g}, 5 \mathrm{~min})$ at $4^{\circ} \mathrm{C}$. The cells were then washed twice in PBS and centrifuged (400 x g, $5 \mathrm{~min})$ at $4^{\circ} \mathrm{C}$. Cells were resuspended in $500 \mu \mathrm{l}$ of binding buffer and $5 \mu \mathrm{l}$ of Annexin V-FITC were added to the samples. The samples were mixed and $5 \mu \mathrm{l}$ of propidium iodide added before being incubated for $15 \mathrm{~min}$ at room temperature in the dark. Cells were then analyzed using a C6 flow cytometer (BD FACS Accuri C6; BD Biosciences).

G-actin/F-actin assay. G-actin and F-actin were detected using the G-actin /F-actin in vivo detection kit (Cytoskeleton, Inc.) according to the manufacturer's instructions. Following $48 \mathrm{~h}$ of treatment with various concentrations of ATL $(0,5,10$ and $20 \mu \mathrm{M}$ ), the cells were lysed with LAS2 buffer (including lysis solution, F-actin stabilization buffer, ATP stock solution and protease inhibitor cocktail stock solution; Cytoskeleton, Inc.) at $37^{\circ} \mathrm{C}$ for $1 \mathrm{~h}$. The cells were isolated by centrifugation at $800 \mathrm{x} \mathrm{g}$ for $5 \mathrm{~min}$ at $4^{\circ} \mathrm{C}$. The cell lysates were then centrifuged at $100,000 \mathrm{x}$ g for $1 \mathrm{~h}$ at $4^{\circ} \mathrm{C}$ and $\mathrm{F}$-actin in the precipitate and $\mathrm{G}$-actin in the supernatant were precipitated. Samples were mixed 5 times with SDS sample buffer and western blot analysis was performed using an anti-actin antibody. Grayscale analysis was performed using ImageJ2x Software (Rawak Software Inc.).

Western blot analysis and immunoprecipitation. Whole-cell, cytoplasmic, nuclear and mitochondrial cytoplasmic proteins from each of the treatment groups were extracted using a corresponding extraction kit. The total protein extractionkit(cat.no.BC3790),cytoplasmic protein extractionkit (cat. no. BC3740), nuclear protein extraction kit (cat. no. R0050) and mitochondrial protein extraction kit (cat. no. SM0020) were purchased from Solarbio Life Sciences, Inc. Equal quantities of protein ( $40 \mu \mathrm{g} /$ lane) were separated by electrophoresis on a $7.5-12 \%$ sodium dodecyl sulfate polyacrylamide, and which were transferred to PVP membranes and detected by specific antibodies targeting the proteins of interest. The membranes were blocked with $5 \%$ non-fat milk for $2 \mathrm{~h}$ at $25^{\circ} \mathrm{C}$. The membranes were incubated with primary antibodies as follows: Cofilin $(1: 1,000)$, p-cofilin $(1: 1,000)$, LIMK1/2 (1:1,000), p-LIMK1/2 (1:1,000), SSH (1:1,000), TESK $(1: 1,000)$, MMP-2/9 $(1: 1,000)$, cytochrome $c(1: 1,000)$ and cleaved caspase-3/9/PARP $(1: 1,000)$ overnight at $4{ }^{\circ} \mathrm{C}$, followed by incubation with anti-rabbit HRP secondary anti-body (1:20,000, cat. no. 7074, Cell Signaling Technology, Inc.), or anti-mouse HRP-conjugated secondary antibody (1:20,000, cat. no. 7076, Cell Signaling Technology, Inc.) for $2 \mathrm{~h}$ at room temperature. $\beta$-actin $(1: 5,000)$, GAPDH $(1: 3,000)$ or Cox IV $(1: 3,000)$ served as the loading controls. Proteins were visualized by exposure to Chem-Doc (Bio-Rad Laboratories, Inc.). The concentration of proteins was determined using a BCA protein detection kit (Beyotime Institute of Biotechnology). All experiments were performed at least in triplicate.

For immunoprecipitation, the cells were dissolved in $1 \%$ NP-40 buffer [50 mM Tris (pH 7.4), $150 \mathrm{mM} \mathrm{NaCl}, 1 \%$ Noindet $\mathrm{P}-40,10 \%$ glycerol, $1 \mathrm{mM}$ PMSF, $10 \mu \mathrm{g} / \mathrm{ml}$ aprotinin, $10 \mu \mathrm{g} / \mathrm{ml}$ leupeptone and $1 \mathrm{mM} \mathrm{Na}_{3} \mathrm{~V}_{4}$. The same amount of protein was then incubated with the primary antibody on a shaking table at $4^{\circ} \mathrm{C}$. Immune complexes were collected with protein $\mathrm{G}$ agarose beads (Santa Cruz Biotechnology Co., Ltd.) and washed several times in lysis buffer. The samples were boiled and western blot analysis was performed as described above.

Immunofluorescence. Cells from each of the treatment groups were grown on chamber slides, collected by centrifugation $(400 \mathrm{x} \mathrm{g}, 5 \mathrm{~min})$ at $4^{\circ} \mathrm{C}$, resuspended gently in pre-warmed $\left(37^{\circ} \mathrm{C}\right)$ staining solution containing $200 \mathrm{nM}$ MitoTracker Red CMXRos (cat. no. M7512, Molecular Probes; Thermo Fisher Scienific, Inc.) for $1 \mathrm{~h}$ at $37^{\circ} \mathrm{C}$, and washed twice with RPMI-1640 medium. This was followed by fixation with $3.7 \%$ of methanol-free formaldehyde for $15 \mathrm{~min}$, and permeabilization with $0.1 \%$ Triton X-100 for $10 \mathrm{~min}$. Slides were blocked with $1 \%$ BSA in PBS for 30 min, then incubated with anti-cofilin (1:50) primary antibody at $4^{\circ} \mathrm{C}$ overnight, followed by the secondary Alexa 488-conjugated goat anti-mouse antibody (1:300, cat. no. R37120, Molecular Probes; Thermo Fisher Scienific, Inc.) for $1 \mathrm{~h}$ at room temperature. Cells were incubated with 50 nM MitoTracker Green FM (cat. no. M7514, Molecular Probes; Thermo Fisher Scienific, Inc.) following fixation. The fluorescent staining of globular and filamentous actin was performed using Fluorescent Deoxyribonuclease I Conjugates (1:500, cat. no. D12371, Molecular Probes; Thermo Fisher Scienific, Inc.) and Fluorescent phallotoxins (1:40, cat. no. R415, Molecular Probes; Thermo Fisher Scienific, Inc.) at $4^{\circ} \mathrm{C}$ overnight, followed by the secondary Alexa 488conjugated goat anti-mouse antibody (1:300, cat. no. R37120, Molecular Probes; Thermo Fisher Scienific, Inc.) for $1 \mathrm{~h}$ at room temperature. The stained samples were mounted with 4',6-diamidino-2-phenylindole (DAPI) at room temperature for $5 \mathrm{~min}$ to counterstain the cell nuclei. Following 5 additional 5-min washes in PBS, the samples were examined under a Leica DM 14000B confocal microscope (Leica Microsystems $\mathrm{GmbH}$ ).

Enzyme-linked immunosorbent assay. The LIMK enzyme was detected using a cell-based fluorometric ELISA kit (ImmunoWay Biotechnology Co., Ltd.) according to the manufacturer's instructions. Cells were seeded onto 96-well plates. Following $48 \mathrm{~h}$ of treatment with various concentrations of ATL $(0,5,10$ and $20 \mu \mathrm{M})$, the cells were fixed by removing the cell growth culture medium, followed by rinsing twice with PBS, and a final incubation with $100 \mu 1$ of $4 \%$ formaldehyde in PBS for adherent cells. Incubation was performed for approximately $30 \mathrm{~min}$ at room temperature. The formaldehyde solution was removed and the cells were rinsed 3 times with wash buffer. The final wash buffer was then removed, and $100 \mu \mathrm{l}$ of Quench buffer was added followed by incubation for $25 \mathrm{~min}$ at room temperature; the plates were sealed and covered with parafilm. The Quench buffer was then removed and the cells were rinsed 3 times for 5 min each with $200 \mu \mathrm{l}$ of wash buffer on a shaker. The wash buffer was removed and $100 \mu \mathrm{l}$ of Blocking Buffer were add followed by incubation for $1 \mathrm{~h}$ at room temperature. After blocking, the plates were washed 3 times with wash buffer for $5 \mathrm{~min}$ each wash. Subsequently, $50 \mu 1$ of Primary Antibody Mixture (1:100 LIMK1/2; 1:100 GAPDH) were added into each relevant 
well on the 96-well plate. The plates were incubated overnight at $4^{\circ} \mathrm{C}$. The plates were sealed with parafilm or incubated in a humid-box in refrigerator, ensuring that the plates were plated at an even level. The Primary Antibody Mixture was then removed, and the wells were washed 3 times for $5 \mathrm{~min}$ each with $200 \mu 1$ of wash buffer with gentle shaking on a shaker. The wash buffer was removed, and $50 \mu 1$ of Secondary Antibody Mixture (DyLight ${ }^{\circledR}$ 649-conjugated anti-rabbit IgG; FITC-conjugated anti-mouse IgG) were then added to each well. The plates were covered and sealed with parafilm, with gentle shaking on a shaker for $1 \mathrm{~h}$ at the room temperature. The Secondary Antibody Mixture was then removed and the cells were washed 3 times for 5 min each time with $200 \mu 1$ of wash buffer. Subsequently, $50 \mu 1$ of $1 \mathrm{X}$ PBS were added to each well on the 96-well plate. The plates were then read at an excitation and emission wavelength: 654/673 (DyLight ${ }^{\circledR} 649$ ) and 495/521 (FITC) and kept in the dark. The fluorescence intensity ratio was calculated between DyLight ${ }^{\circledR} 649$ vs. FITC, comparing the ratio before and after treatment.

Animal experiments. U87MG cells were trypsinized and resuspended in PBS at a density of $1 \times 10^{7} / \mathrm{ml}$. Under aseptic conditions, $100 \mu 1$ of cell suspension was subcutaneously injected near the axillary fossa of male nude mice (BALB/c nu/nu, 4 weeks old, weighing 18-19 g, 25 mice in total. The mice were maintained in a specific pathogen-free grade animal facility on a $12 \mathrm{~h} \mathrm{light/dark} \mathrm{cycles} \mathrm{at} 25 \pm 2^{\circ} \mathrm{C}$. The mice were provided with free access to sterilized food and water and were allowed to acclimatize for 7 days before start of the experiment. All procedures were performed at the SPF Laboratory Animal Center of Dalian Medical University, Dalian, China).

Following injection, the weights of the mice were recorded daily, and the longest and shortest diameters of the tumors were measured using a Vernier caliper. When the tumors reached a size of $3 \times 4 \mathrm{~mm}$, the mice were randomly divided into 3 experimental groups with 5 mice in each group. In group A, the animals were injected with $100 \mu 1 \mathrm{PBS}$; in group B, the animals were treated with a low dose of ATL $(10 \mathrm{mg} / \mathrm{kg}$ in PBS containing 33\% propylene glycol, $100 \mu 1$ ); and group C, the animals were treated with a high dose of ATL $(20 \mathrm{mg} / \mathrm{kg}$ in PBS containing 33\% propylene glycol, $100 \mu \mathrm{l}$ ). The doses used were selected with reference to previous research (15). Treatments were delivered by intraperitoneal injection once a day for 15 days. All experimental animals were sacrificed and tumor tissues were collected for immuno-histochemical staining and western blot analysis. All procedures were in performed in line with the National Institutes of Health Guidelines for the Care and Use of Laboratory Animals (National Institutes of Health, Bethesda, MD, USA). The experimental protocol was approved by the Animal Care and Ethics Committee of Dalian Medical University.

Statistical analysis. A Student's t-test (two-tailed), a t-test with Welch's correction, and an F-test were used to statistically analyze the datasets using GraphPad Prism 6.0 software (GraphPad Software, Inc.). The concrete methods of t-test analysis in the study were as follows: The data of 2 groups for comparison were analyzed using an F-test firstly (homogeneity test of variance). When the value of the F-test was $>0.05$, the value of the t-test was obtained according to the heteroscedasticity double sample test. When the value of the F-test was $<0.05$, the value of the $t$-test was obtained according to the heteroscedasticity double sample test. A value from the t-test $<0.05$ indicated significant differences between the 2 experimental groups and a value of the t-test $>0.05$ indicated no statistically significant differences between the 2 experimental groups. Two-way analysis of variance (ANOVA) followed by a Bonferroni's test for multiple comparisons were performed to analyze the data involving multiple groups. The data are represented as the means $\pm \mathrm{SD}$ of at least 3 independent experiments. P-values $<0.05$ were considered to indicate a statistically significant difference. SPSS 18.0 software (SPSS Inc.) was used for all of the statistical analyses.

\section{Results}

ATL induces the dephosphorylation of Cofilin and regulates the ratio of G/F-actin. A previous study by the authors (6) demonstrated that ATL significantly reduced the migration and invasiveness of GBM cells. Moreover, previous research has indicated that the G/F-actin ratio is an indicator of actin dynamics and is responsible for regulating cell migration and invasion (16). Cofilin is a binding protein of actin that functions function of cofilin is to regulate the polymerization and depolymerization of actin. Phosphorylated cofilin is inactive which hinders the process of F-actin depolymerization into G-actin, and disrupts the formation of invasive pseudopodia to enhance the migration and invasion of tumor cells $(11,17)$.

In the present study, G-actin was first separated from F-actin using a G/F-actin separation kit. The results of western blot analysis revealed that ATL significantly increased the expression of G-actin and reduced the expression of F-actin (Fig. 1B). Similarly, it was also confirmed that ATL significantly upregulated the expression of G-actin and downregulated the expression of F-actin in a concentration-dependent manner, as shown by immunofluorescence microscopy confocal experiments (Fig. 1C).

Subsequently, whole-cell protein, as well as cytoplasmic and mitochondrial proteins were isolated from the U87MG and U251 cells and were subjected to western blot analysis (Fig. 2A-D). In the whole-cell extract, it was found that ATL significantly inhibited the expression of p-cofilin in a concentration-dependent manner, whilst the total level of cofilin was not markedly altered (Fig. 2A). These results indicated that ATL can induce the dephosphorylation and activation of cofilin and depolymerize F-actin into G-actin, suggesting a potential role of ATL in inhibiting the migration and invasion of GBM cells.

ATL induces cofilin and G-actin co-translocation to the mitochondria. The authors have previously demonstrated that ATL induces the release of cytochrome $c$ from the mitochondria into the cytoplasm through endogenous pathways to initiate the caspase cascade pathway and induce apoptosis in GBM cells (6). Previous research has found that cofilin can translocate to the mitochondria to increase the permeability transition pores, release cytochrome $c$ and initiate the apoptotic cascade (18). In the present study, it was found that 


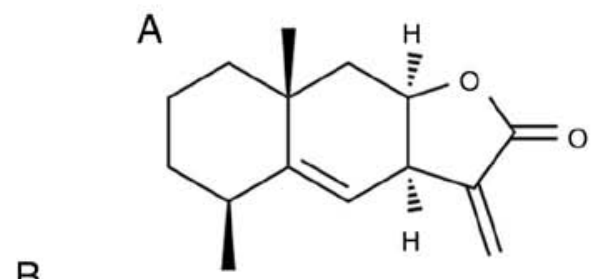

B
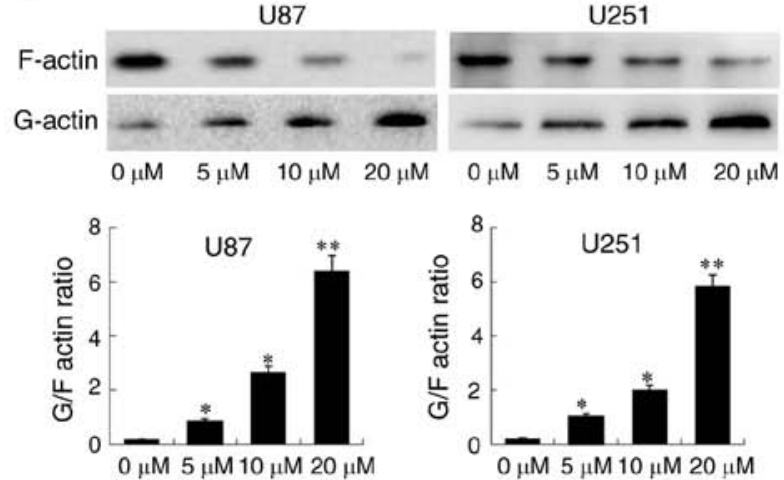

C
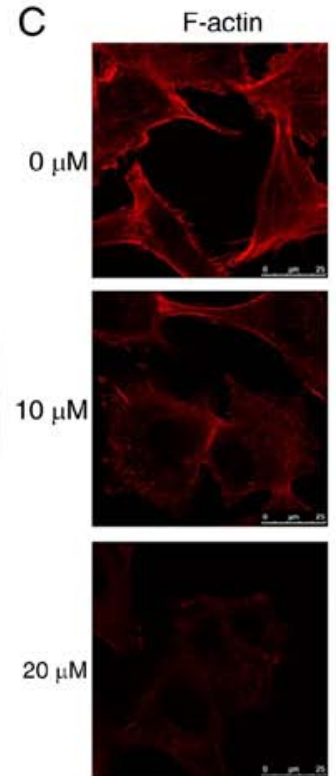

G-actin
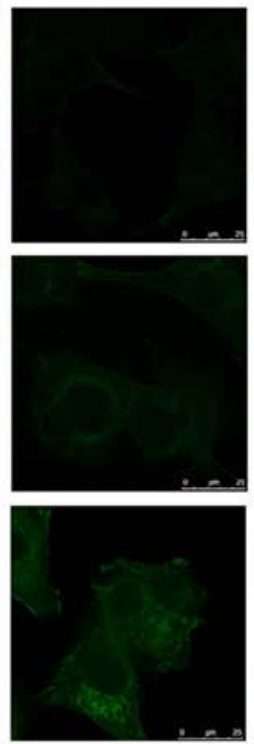
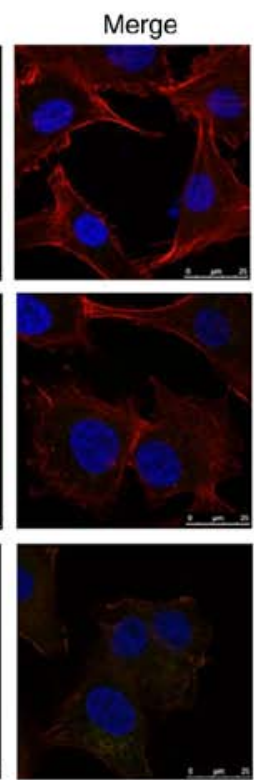

Figure 1. ATL regulates the ratio of G/F-actin. (A) Chemical structure of ATL. Molecular formula, $\mathrm{C}_{15} \mathrm{H}_{20} \mathrm{O}_{2}$. Molecular weight, 232.32 g/mol. (B) At 48 h following treatment of the U87MG and U251 cells with ATL $(0-20 \mu \mathrm{M})$, the levels of F-actin and G-actin were measured using the G-actin/F-actin assay kit. (C) The levels of F-actin (red) and G-actin (green) in U87MG cells treated with ATL were imaged by confocal microscopy. ${ }^{*} \mathrm{P}<0.05$ and ${ }^{* *} \mathrm{P}<0.01$ vs. the DMSO group. ATL, alantolactone.

the expression of cofilin in the cytoplasm was significantly decreased (Fig. 2B) and the expression in the mitochondria was significantly increased (Fig. 2C). The expression of Cofilin in whole cells was not significantly changed (Fig. 2A) after ATL treatment. These observations indicate that ATL can promote the transfer of cofilin from the cytoplasm to the mitochondria.

It was also found that the expression of p-cofilin (Ser 3) protein was significantly decreased in whole cells and in the cytoplasm (Fig. 2B), whilst its expression was not detected in the mitochondria (Fig. 2C). These results confirm that cofilin may be activated by dephosphorylation at the Ser 3 site and only activated cofilin may be translocated into the mitochondria, whilst p-Cofilin cannot undergo mitochondrial translocation. Cofilin (red light) and mitochondria (green light) were stained with specific fluorescence probes observed under a laser confocal microscope (Fig. 3A). Following treatment with ATL, the fluorescence of cofilin and the mitochondria overlapped (red light and green light overlapped into yellow light), further confirming that ATL induced the translocation of cofilin to the mitochondria.

Following treatment with ATL, the expression of actin was upregulated in the mitochondria (Fig. 2C) and downregulated in the cytoplasm (Fig. 2B) in a concentration-dependent manner. The expression of actin at the whole cell protein level was not significantly altered (Fig. 2A). These data suggest that ATL can significantly increase the ratio of G-actin to F-actin; thus, it was hypothesized that G-actin is transferred to the mitochondria. Subsequently, specific fluorescence staining technology was used to observe the localization associations between G-actin, F-actin and the mitochondria under a laser confocal microscope to examine the hypothesis. The results revealed that the fluorescence of G-actin and the mitochondria overlapped (yellow light, Fig. 3B) whilst the co-localization between F-actin and the mitochondrial did not occur (Fig. 3C), confirming the hypothesis.
We speculate that the ATL-induced translocation of Cofilin to the mitochondria causes apoptosis and may also be related to actin translocation to mitochondria. We conducted immunoprecipitation experiments to detect the levels of correlation between Cofilin and actin. As shown in Fig. 2D, we found that the direct binding of Cofilin and actin in mitochondrial components was significantly increased after treated with ATL. Also, Cofilin (red light) and G-actin (green light) were found to be co-localized and had overlapping fluorescence (yellow light) detected by confocal observation (Fig. 3D). These results confirm that ATL induces cofilin and G-actin to co-translocate to the mitochondria. These proteins interact with each other and mediate the mitochondrial/cytochrome $c$ pathway and to initiate the caspase cascade signaling pathway, leading to apoptosis.

ATL inhibits the activity of the LIMK enzyme. Studies have demonstrated that the activity of cofilin is mainly regulated by phosphorylation and dephosphorylation, whilst the third serine site of Cofilin is only one phosphorylation site (19). When the serine 3 site is phosphorylated, cofilin loses its activity and F-actin will accumulate due to blocked depolymerization. By contrast, Cofilin will regain the function of depolymerizing F-actin after the serine 3 site is dephosphorylated. The enzymes that catalyze cofilin phosphorylation are LIMK and TESK, whilst the enzymes that can remove cofilin phosphorylation include SSH (20).

To further explore the target of ATL in regulating cofilin activity, the expression of phosphorylase and dephosphorylase that catalyze cofilin were detected. As shown in Fig. 4A, ATL significantly inhibited the expression of p-LIMK1/2 in a concentration-dependent manner, whilst the total levels of LIMK1 and LIMK2, TESK and SHH were not markedly altered. Furthermore, a significant downward trend of p-LIMK1/2 protein was detected by ELISA (Fig. 4B). Based 

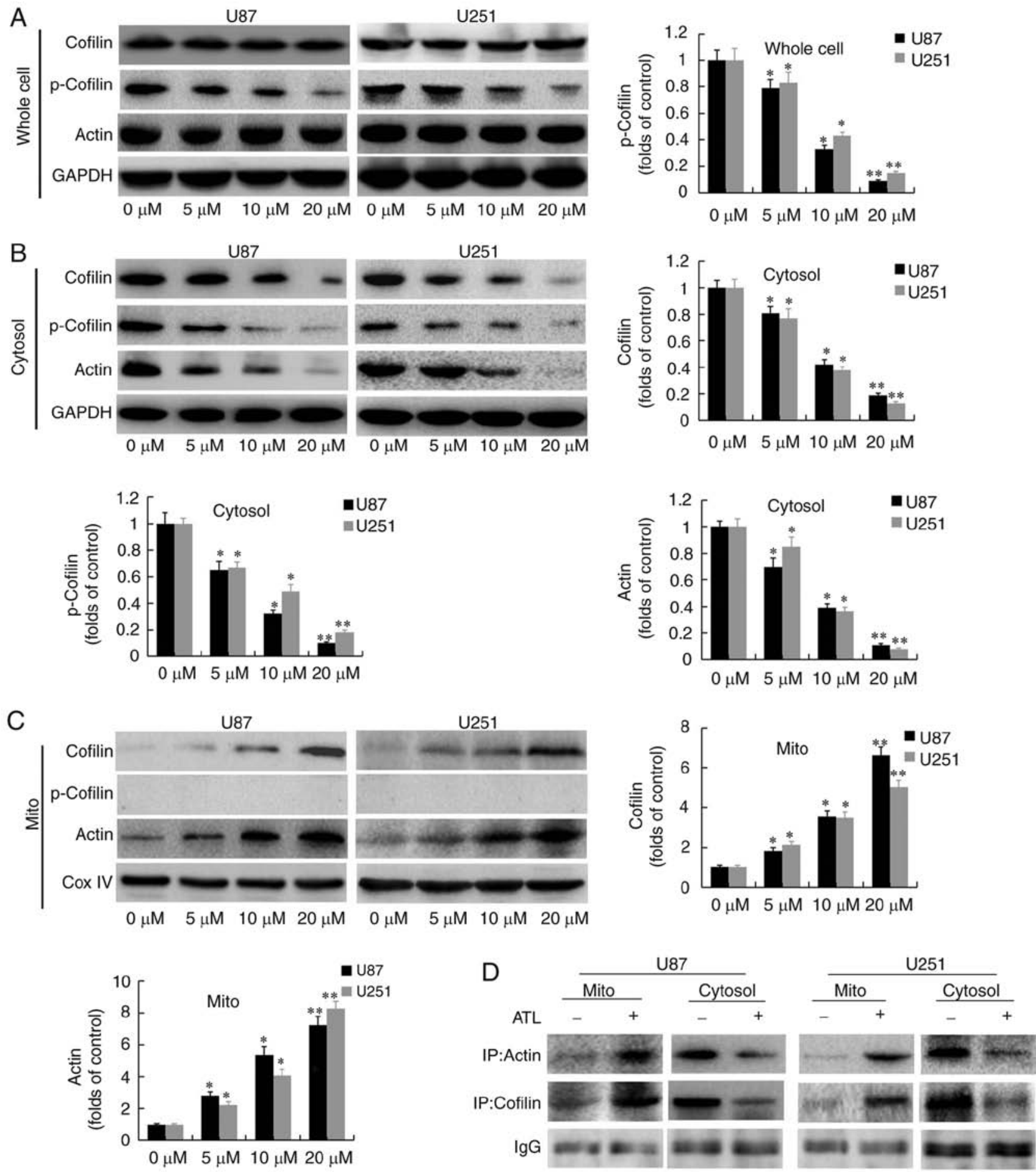

Figure 2. ATL induces cofilin and G-actin co-translocation to the mitochondria. After U87MG and U251 cells were treated with various concentrations of ATL $(0-20 \mu \mathrm{M})$ for $48 \mathrm{~h}$, whole-cell, cytosolic and mitochondrial lysates were prepared. (A) Western blot analysis was performed using antibodies against cofilin, p-cofilin, actin and GAPDH. (B) The cytosolic fractions were subjected to western blot analysis using antibodies against cofilin, p-cofilin, actin and GAPDH. (C) The mitochondrial fractions were subjected to western blot analysis using antibodies against cofilin, p-cofilin, actin and Cox IV. (D) Cytosolic and mitochondrial fractions of control and ATL-treated cells were prepared and subjected to immunoprecipitation using an anti-cofilin antibody, followed by western blot analysis. ${ }^{*} \mathrm{P}<0.05$ and $^{* *} \mathrm{P}<0.01$ vs. the DMSO group. ATL, alantolactone; Cox IV, cytochrome $c$ oxidase.

on these data, it was hypothesized that ATL may activate cofilin by inducing the dephosphorylation of LIMK, inhibiting the activity of LIMK enzyme and blocking the phosphorylation of cofilin.

ATL inhibits the migratory ability and invasiveness of GBM by targeting LIMK enzyme activity. In a previous study, the authors demonstrated that ATL suppressed the migratory ability and invasiveness of GBM cells and also downregulated the expression of MMP-2 and MMP-9 (6). Thus, in the present study, experiments were conducted to verify whether ATL targeting LIMK enzyme activity during the activation of cofilin would inhibit the migration and invasiveness, and induce the apoptosis of GBM cells. U87MG cells were 

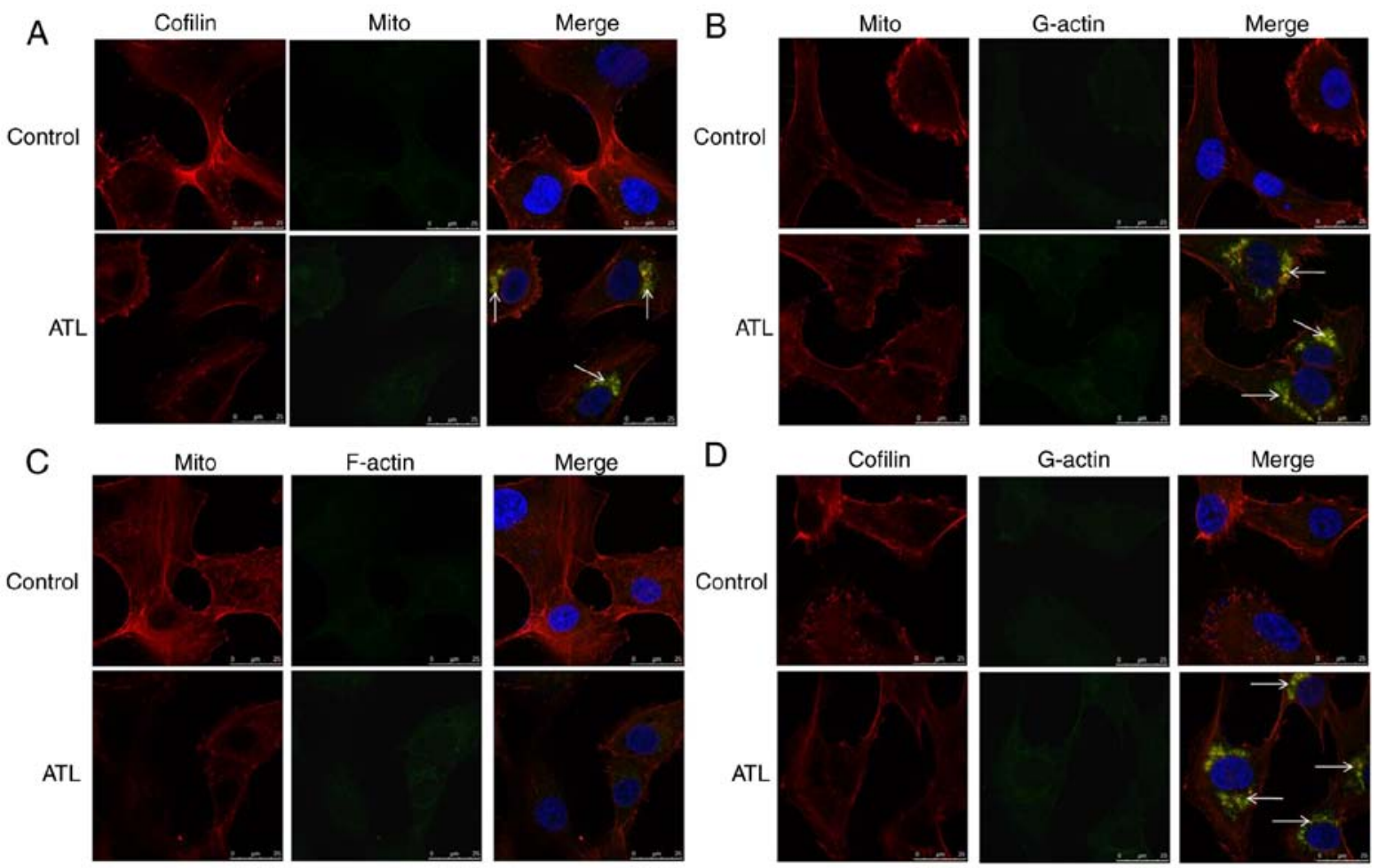

Figure 3. (A) The levels of cofilin (red) and mitochondria (green) in U87MG cells treated with ATL were imaged by confocal microscopy analysis. White arrows indicate overlapping parts (yellow). (B) Levels of G-actin (green) and mitochondria (red) in U87MG cells treated with ATL were imaged by confocal microscopy. White arrows indicate overlapping parts (yellow). (C) The levels of F-actin (green) and mitochondria (red) in U87MG cells treated with ATL were imaged by confocal microscopy. (D) The levels of Cofilin (red) and G-actin (red) in U87MG cells treated with ATL were imaged by confocal microscopy. White arrows indicate overlapping parts (yellow). ATL, alantolactone.

A
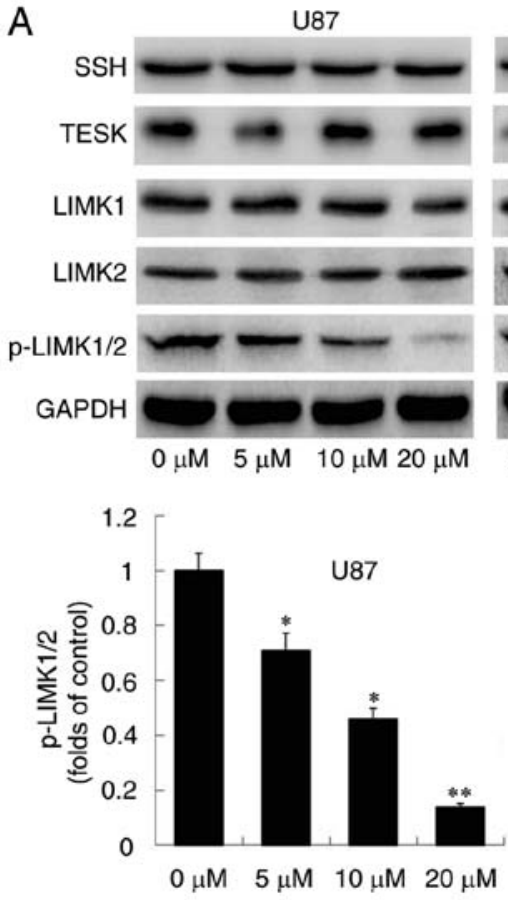

U251

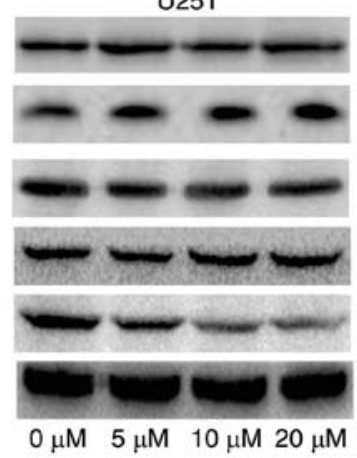

B

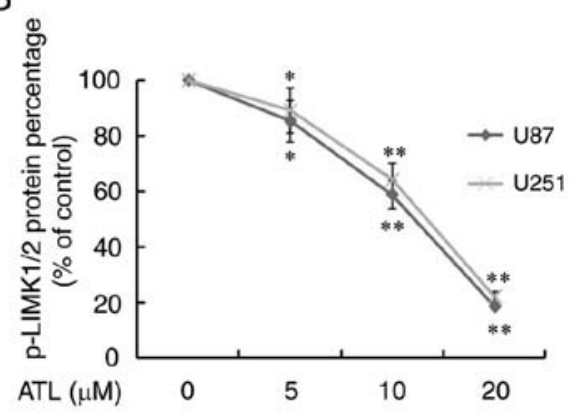

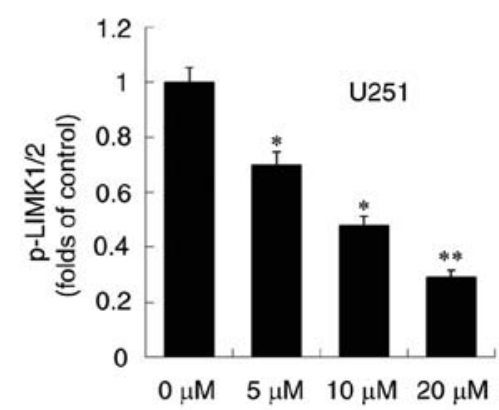

Figure 4. ATL inhibits the activity of LIMK enzyme. At $48 \mathrm{~h}$ following treatment of U87MG and U251 cells with ATL, (A) the protein levels of the SHH, TESK, LIMK1, LIMK2, p-LIMK1/2 and GAPDH were detected by western blot analysis, and (B) the percentage p-LIMK1/2 protein was detected by ELISA. "P<0.05 and ${ }^{* * *} \mathrm{P}<0.01$ vs. the DMSO group. ATL, alantolactone; LIMK, LIM kinase; SSH, Sonic hedgehog; TESK, testis associated actin remodelling kinase.

pre-treated with LIMKi $3(5 \mu \mathrm{M})$ for $8 \mathrm{~h}$, and then ATL $(10 \mu \mathrm{M})$ was added at the corresponding time points to detect migration and invasion. LIMKi 3 is a specific selective LIMK inhibitor. The results of the wound scratch (Fig. 5A and C) and invasion assays (Fig. 5B and D) revealed significant differences between the ATL, LIMKi 3 and ATL + LIMKi 3 groups 

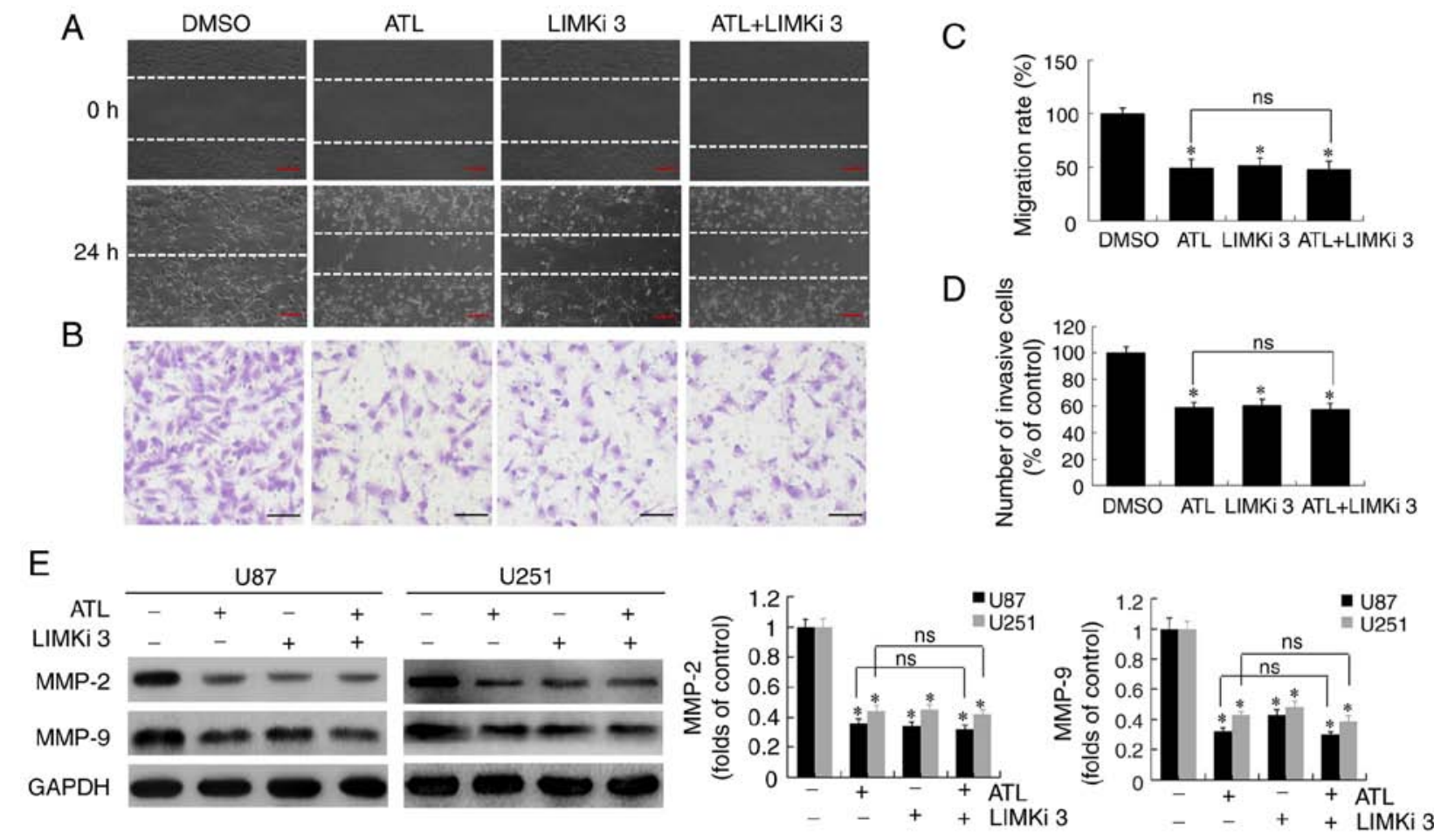

Figure 5. ATL inhibits the migratory ability and invasiveness of GBM cells by targeting LIMK enzyme activity. (A) Wound healing test results (scale bar, $100 \mu \mathrm{m}$ ) and (C) mobility calculation results of the U87MG cells; (B) Transwell assay results (scale bar, $50 \mu \mathrm{m}$ ) and (D) the number of invasive cells (\% of control) of the U87MG cells. (E) The protein expression of MMP-2 and MMP-9 was detected by western blot analysis of the U87MG and U251 cells. Similar tests were repeated at least 3 times. ${ }^{*} \mathrm{P}<0.05$ vs. the DMSO group; ns, not significant ( $>0.05$, the ATL group vs. the ATL + LIMKi 3 group). GBM, gliobastoma; ATL, alantolactone; LIMK, LIM kinase; MMP, matrix metalloproteinase.

compared to the control group $(\mathrm{P}<0.05)$. No significant differences were observed between the ATL and ATL + LIMKi 3 group $(\mathrm{P}>0.05)$. The above-mentioned results indicated that ATL, similar to LIMKi, specifically inhibited LIMK enzyme activity to suppress the migratory ability and invasiveness of GBM cells.

The U87MG and U25 cells were then treated in the same manner and the protein expression of MMP-2 and MMP-9 was detected by western blot analysis. The results revealed (Fig. 5E) that the expression of these 2 proteins was differed significantly between the ATL, LIMKi 3 and ATL + LIMKi 3 groups, and the control group. No significant difference was observed between the ATL and ATL + LIMKi groups. These data suggest that ATL may play an anti-migratory and anti-invasive role in GBM by inhibiting LIMK enzyme activity, activating cofilin, and downregulating MMP-2 and MMP-9 protein expression.

ATL induces the apoptosis of GBM cells by targeting LIMK enzyme activity. Previously, it was confirmed that ATL induces the release of cytochrome $c$ from the mitochondria into the cytoplasm through endogenous pathways to initiate the caspase cascade pathway, and induces the apoptosis of GBM cells (6). In the present study, U87MG cells were pre-treated with LIMKi 3 for $8 \mathrm{~h}$ and ATL was then applied $(10 \mu \mathrm{M})$ for $24 \mathrm{~h}$ before detecting apoptosis by flow cytometry. As shown in Fig. 6A and B, compared to the control group, the number of apoptotic cells in the ATL, LIMKi 3 and ATL + LIMKi 3 groups increased significantly $(\mathrm{P}<0.05)$. No significant difference in the levels of apoptosis was observed between the ATL and ATL + LIMKi 3 groups $(\mathrm{P}>0.05)$.
The expression of key proteins regulating apoptosis was then detected by western blot analysis. As shown in Fig. 6C, the expression levels of cleaved caspase-3, cleaved caspase-9, cleaved PARP and cytochrome $c$ were significantly increased in the ATL, LIMKi 3 and ATL + LIMKi 3 groups compared to the control group $(\mathrm{P}<0.05)$. No significant differences in the expression of apoptosis-associated proteins were detected between the ATL and ATL + LIMKi 3 groups ( $>0.05)$. These results confirm that ATL can activate cofilin by targeting LIMK enzyme activity. In addition, cofilin and G-actin co-translocate to the mitochondria, causing mitochondrial damage and the release of cytochrome $c$ from the mitochondria to the cytoplasm, which acts to initiate the caspase signaling pathway to induce apoptosis.

ATL inhibits the expression of p-cofilin and p-LIMK1/2 in a heterotopic xenograft tumor model. To verify the results from the cell experiments, in vivo experiments by were conducted establishing a GBM xenograft model. Previously, it was confirmed that ATL can inhibit the growth of transplanted tumors in nude mice in a dose-dependent manner (6). In the present study, transplanted tumor tissues from each of the experimental groups were first examined by immunohistochemical analysis. The results revealed that ATL inhibited the expression of p-cofilin in a dose-dependent manner (Fig. 7A). Western blot analysis also revealed that ATL significantly downregulated the expression levels of p-cofilin and p-LIMK1/2 in tumor tissues in vivo (Fig. 7B).

Molecular mechanisms responsible for the inhibitory effects of ATL on the GBM metastatic phenotype and the induction 

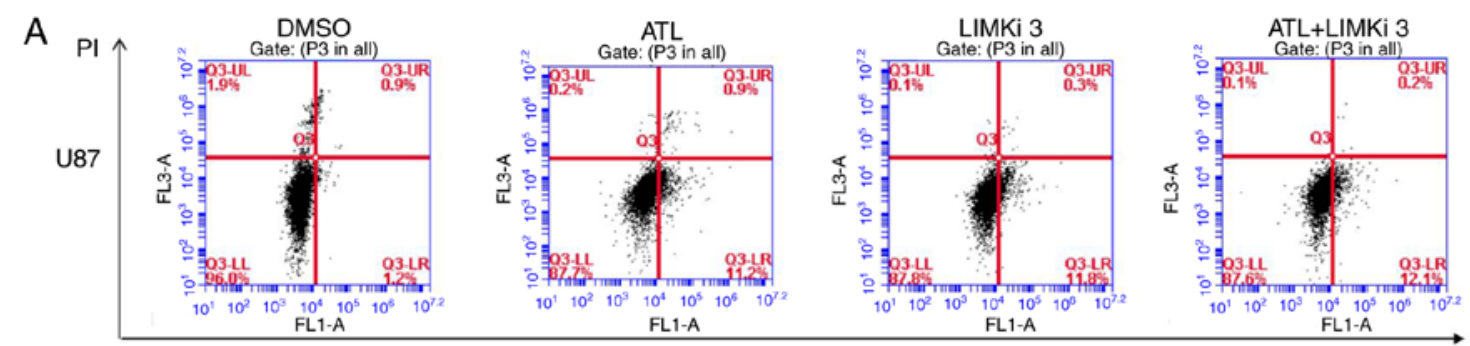

Annexin V-PITC
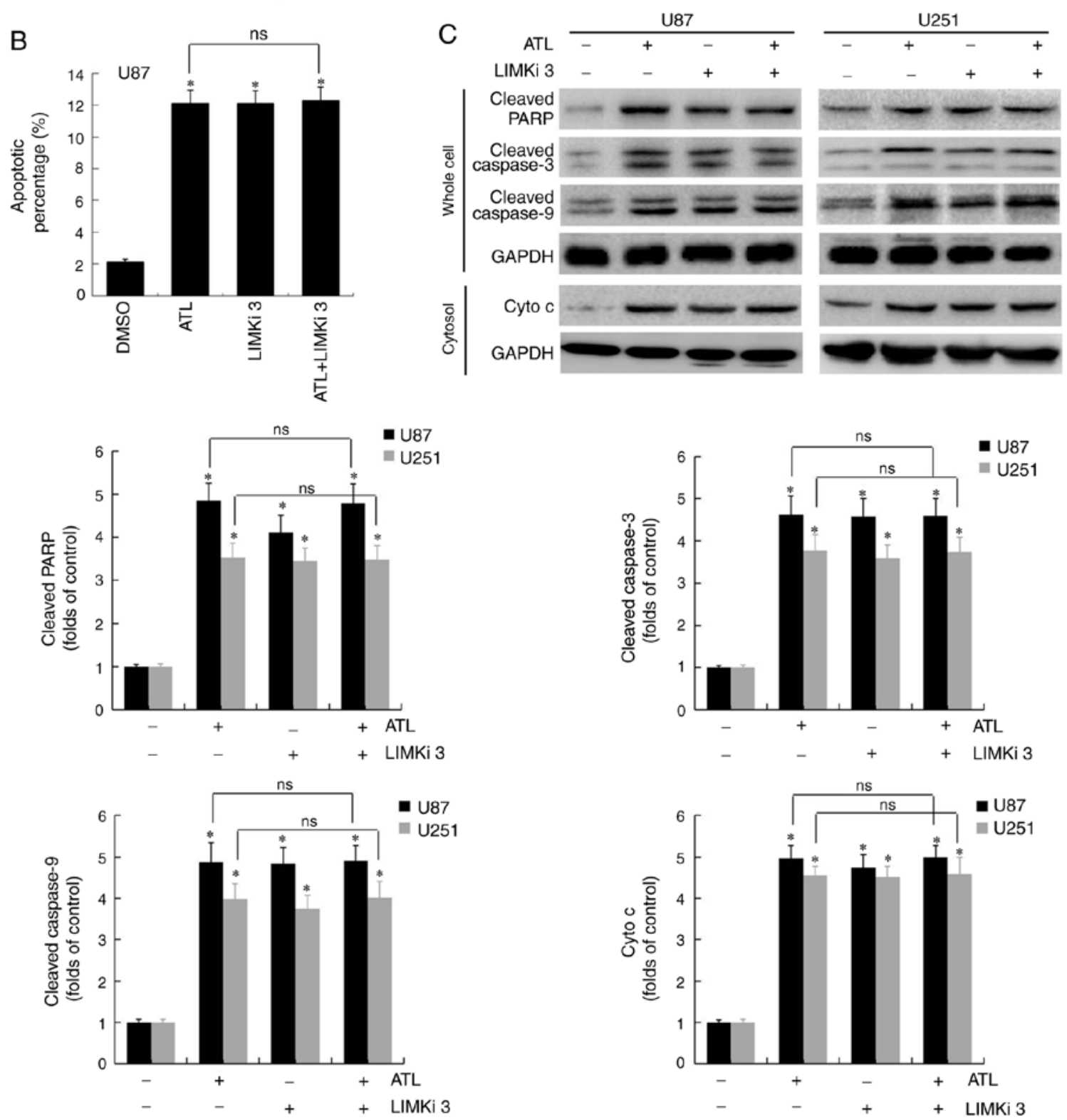

Figure 6. ATL induces the apoptosis of GBM cells by targeting LIMK enzyme activity. (A) Detection results of apoptotic cells and (B) calculation results of the apoptotic rate. (C) Protein expression levels of cleaved caspase-3, cleaved caspase-9, cleaved PARP and cytochrome $c$ in the U87MG and U251 cells were detected by western blot analysis. Similar tests were repeated at least 3 times. "P $<0.05$ vs. the DMSO group; ns, not significant (P>0.05, the ATL group vs. the ATL + LIMKi 3 group). GBM, gliobastoma; ATL, alantolactone; LIMK, LIM kinase.

of apoptosis. As demonstrated by the above-mentioned results, ATL may activate cofilin through the targeted inhibition of LIMK enzyme activity. It can upregulate the ratio of G/F actin, and inhibit the migration and invasion of GBM cells. Activated cofilin and G-actin can be co-transferred to the mitochondria to initiate the mitochondrial/cytochrome $c$ pathway and induce apoptosis (Fig. 8).

\section{Discussion}

GBM is the most common aggressive brain tumor and has an extremely poor prognosis as current standard of care treatments lack efficacy. There is an urgent need for the development of novel therapeutics that are effective against the disease. With advances in targeted therapies, it is particularly important to 


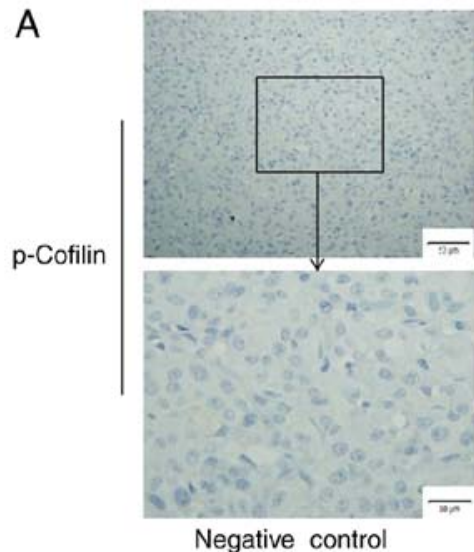

\section{B}

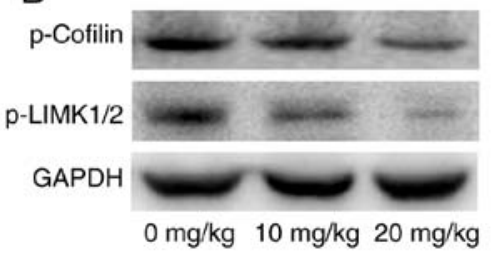

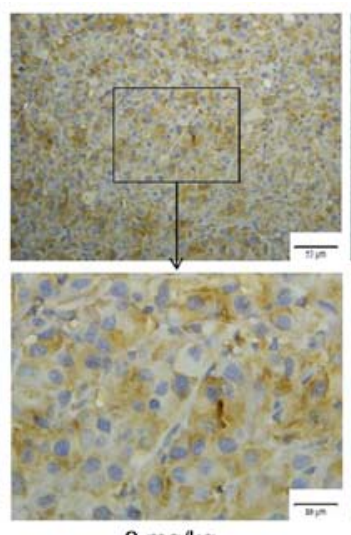

$0 \mathrm{mg} / \mathrm{kg}$

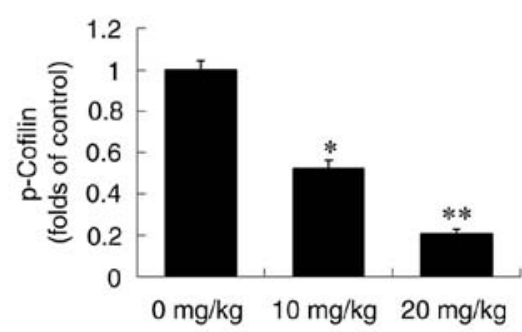

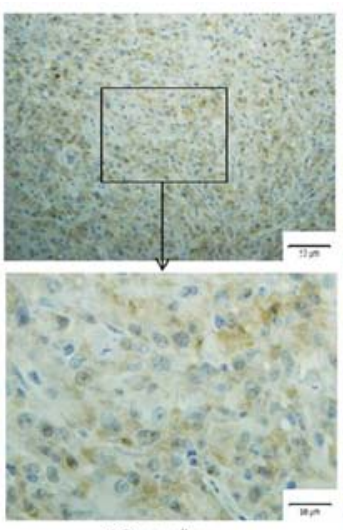

$10 \mathrm{mg} / \mathrm{kg}$

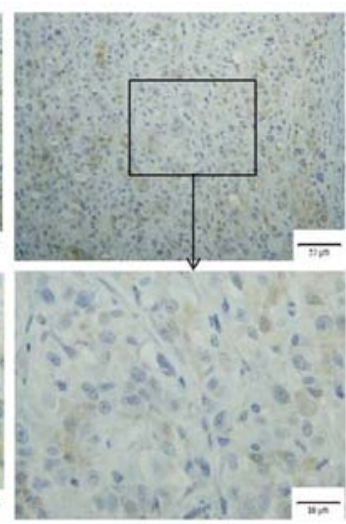

$20 \mathrm{mg} / \mathrm{kg}$

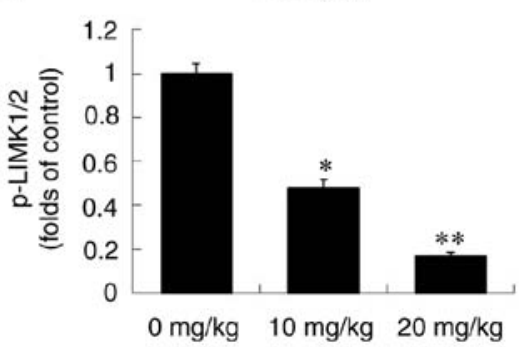

Figure 7. ATL inhibits the expression of p-cofilin and p-LIMK1/2 in a heterotopic xenograft tumor in nude mice. (A) Protein expression of p-cofilin in tumor samples was examined by immunohistochemical analysis (scale bar, 50 and $10 \mu \mathrm{m}$ ). (B) Protein expression of p-cofilin and p-LIMK1/2 was detected in tumor samples by western blot analysis. ${ }^{*} \mathrm{P}<0.05$ and ${ }^{* *} \mathrm{P}<0.01$ vs. the $0 \mathrm{mg} / \mathrm{kg}$ group. ATL, alantolactone; LIMK, LIM kinase.

explore new targets for the diagnosis and treatment of GBM. ATL is a small molecule natural compound that has a variety of pharmacological activities including antitumor effects (21-23). In a previous study by the authors (6), it was found that ATL inhibited the proliferation, growth, migration and invasion of GBM cells, and induced apoptosis. The present study aimed to systematically examine the specific molecular mechanism and regulatory targets of ATL in GBM.

The migration and invasion of tumor cells is a complex process (24) that consists of 4 basic steps. The process begins with the separation of cancer cells from tumor entities. The second step involves adhesion to extracellular matrix followed by the activation of matrix metalloproteinases (typically MMP-2, MMP-9) to degrade the extracellular matrix and the final involves complete cell movement and contraction. The migratory movement of cells is the first core step in which actin and its regulatory proteins play a central role (25). Actin can be divided into 2 forms, fibrous F-actin (polymer) and spherical $\mathrm{G}$-actin (monomer). These forms exist in the form of dynamic equilibrium by depolymerizing F-actin into G-actin and polymerizing G-actin into F-actin. Actin is closely related to tumor cell movement, vascular invasion and metastasis. Tumor cells break the dynamic balance of actin by blocking the depolymerization of F-actin. This causes the accumulation of F-actin that induces the formation of invasive pseudopodia of tumor cells under the guidance of chemotactic signals and promotes the migration, invasion and metastasis of tumor cells (26).

Actin depolymerizing factors are a group of proteins that can bind to actin. Cofilin is an important family member with a molecular weight of $21 \mathrm{kDa}$ and is expressed in all eukaryotic cells. Cofilin and contains sites that can bind to actin at both ends of protein $\mathrm{N}$ and $\mathrm{C}$ termini (20). Previous research has demonstrated that cofilin plays a key role in regulating the speed and direction of tumor cell migration and in mediating adhesion to the extracellular matrix during invasion and metastasis (27). Activated cofilin can combine with actin to depolymerize F-actin in the multimeric form into G-actin in monomer form, increasing the conversion of actin. Therefore, cofilin is a key molecule that regulates dynamic changes in the actin cytoskeleton (28). The activation or inactivation of cofilin is closely related to the invasion and metastasis of tumor cells.

The n-terminal serine 3 site of cofilin is the only phosphorylation site in its structure. The phosphorylation and dephosphorylation of cofilin are closely related to the assembly, decomposition and cleavage of actin (9). Once the Ser 3 site is phosphorylated, cofilin will lose its activity and cannot combine with $\mathrm{F}$-actin to prevent $\mathrm{F}$-actin from depolymerizing into $\mathrm{G}$-actin and induce tumor cell migration, invasion and metastasis (29).

It has been demonstrated that the enzymes that catalyze cofilin phosphorylation are LIMK and TESK. The phospholipase that removes cofilin phosphorylation is $\mathrm{SSH}$. LIMK is a key enzyme that regulates cofilin (19). LIMK is a serine/threonine protein kinase that exists in eukaryotes. It has 3 highly related family members, LIMK1 and LIMK2. Phosphorylated LIMK is an activated form and can specifically catalyze the phosphorylation of the Ser 3 site of cofilin that inactivates cofilin. The change of cofilin activity regulated by LIMK is directly related to the invasion, movement and metastasis of tumor cells (30).

Previously, it was confirmed that ATL can suppress the migratory ability and invasiveness of GBM cells and downregulates the expression of MMP-2 and MMP-9 (6). However, in the present study, it was found that the expression of $\mathrm{p}$-cofilin was significantly decreased, and the ratio of $\mathrm{G} / \mathrm{F}$-actin was significantly increased by ATL. It was found that ATL specifically downregulated the expression of $\mathrm{p}$-LIMK $1 / 2$ in the 


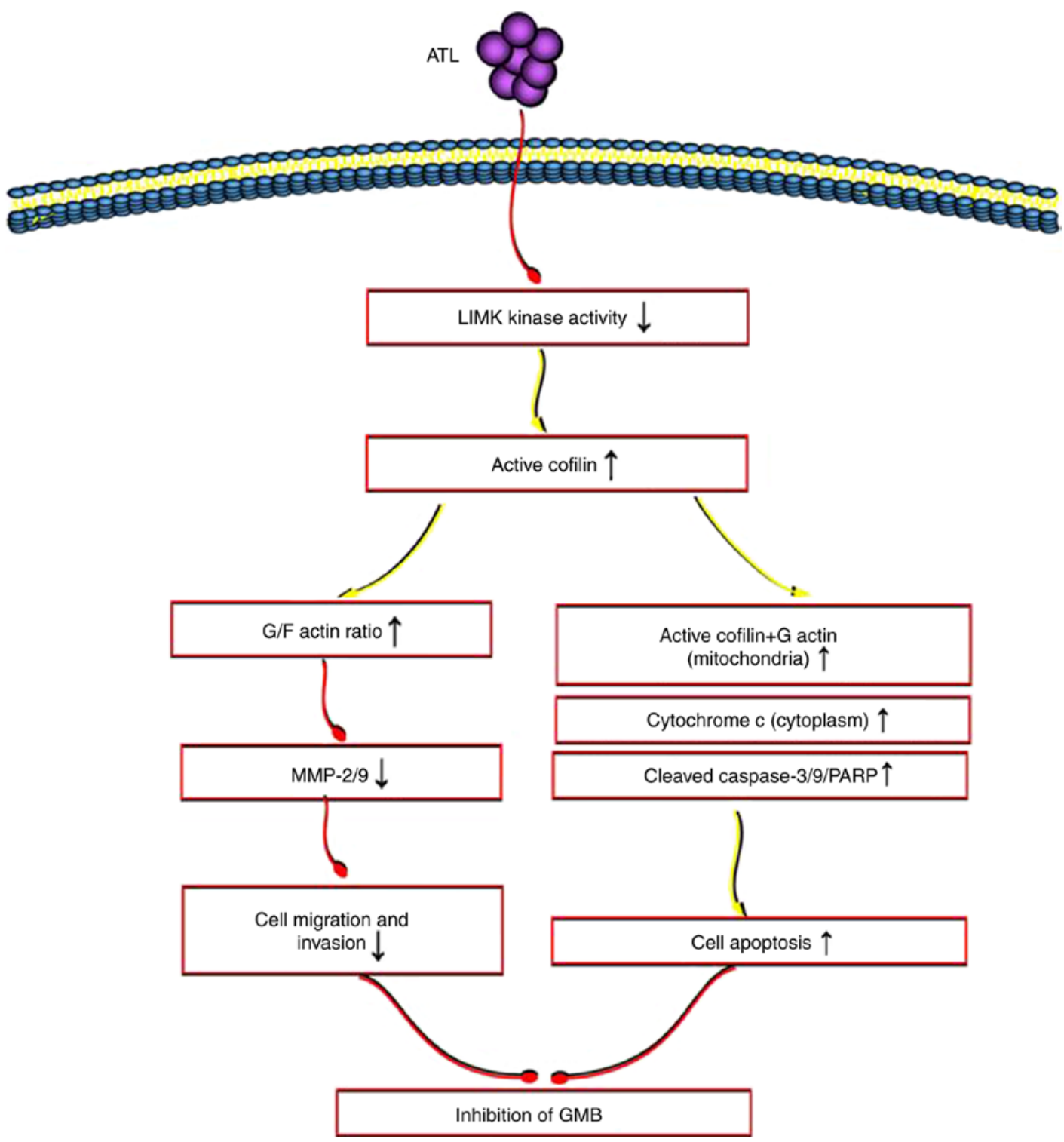

Figure 8. Schematic diagram of the molecular mechanisms of the effects of ATL against GBM. ATL may activate cofilin through targeted inhibition of LIMK enzyme activity. It can up-regulate the ratio of G/F actin and inhibit the migration and invasion of GBM cells. Activated cofilin and G-actin can be co-transferred to the mitochondria to initiate mitochondrial/cytochrome c pathway and induce apoptosis. GBM, gliobastoma; ATL, alantolactone; LIMK, LIM kinase; MMP, matrix metalloproteinase.

detection of cofilin phosphorylation and dephosphorylation enzyme. The protein expression of MMP-2/MMP-9 and the characterization of migration and invasion were determined following treatment with LIMKi (a positive inhibitor of the LIMK enzyme). It was confirmed that ATL inhibited migration and invasion by inhibiting LIMK enzyme activity and activating cofilin. These changes resulted in an increased ratio of $\mathrm{G} / \mathrm{F}$-actin. In addition, it was verified that ATL significantly reduced the expression of $\mathrm{p}$-LIMK $1 / 2$ and $\mathrm{p}$-cofilin in vivo using a xenograft tumor model established in nude mice.

Apoptosis is the process of programmed cell death and the unlimited growth of tumor cells results from the inhibition of apoptosis. Studies have found that ATL can induce the apoptosis of liver, colon, lung and leukemia cells (31-34). The occurrence of apoptosis is mainly divided into the endogenous and exogenous pathways, of which the endogenous pathway is also known as the mitochondrial-cytochrome $c$ pathway (35). In a previous study, the authors confirmed that ATL can promote the transmission of cytochrome $c$ from the mitochondria to the cytoplasm and initiate the caspase cascade to induce the apoptosis of GBM cells (6).

The mechanism through which ATL regulates the transmission of cytochrome $c$ by permeability changes caused by mitochondrial damage remains largely unknown. Studies have found that the mitochondrial translocation of cofilin is an early step in the induction of apoptosis. Moreover, it has been confirmed that only activated cofilin (dephosphorylated state) can be transferred to the mitochondria and that this transfer is enabled by combining the carboxyl-terminal sequence of the target signal with the amino-terminal of the mitochondria (36). A previous study found that following the activation of cofilin dephosphorylation Ser 3 site, activated Cofilin translocated to the outer mitochondrial membrane to open the permeability transition pore and induced the release of cytochrome $c$ to the cytoplasm, leading to cell apoptosis (37). In the present study, it was found that following treatment with ATL, p-Cofilin 
was not detected in the mitochondria. The expression of active cofilin was significantly increased in the mitochondria and significantly decreased in the cytoplasm. In addition, the co-localization of cofilin in the mitochondria was further confirmed, indicating that ATL can induce active cofilin to translocate to the mitochondria.

As previously demonstrated, the mitochondrial translocation of cofilin is highly dependent on the mitochondrial translocation of actin (38). However, the mechanism of interaction between cofilin and actin translocation remains unclear. In the present study, it was demonstrated that ATL upregulated actin in the mitochondria, whilst actin expression in the cytoplasm decreased. Moreover, only G-actin was colocalized in the mitochondria, whilst F-actin was not. It was found that Cofilin and actin exhibited an obvious co-precipitation in the mitochondria following treatment with ATL, which only minimally occurred in the cytoplasm. It was also confirmed that only G-actin co-localized with cofilin.

It was hypothesized that ATL can promote cofilin and G-actin co-translocation to the mitochondrial outer membrane to initiate the endogenous apoptosis pathway. To examine this hypothesis, LIMKi was used to detect the changes in the number of apoptotic cells and the expression of key proteins in the cytochrome $c$ and caspase cascade pathways. The results verified that ATL at least partially induced apoptosis by inhibiting LIMK enzyme activity, activating cofilin, inducing the co-translocation of activated cofilin and G-actin to the mitochondria. These changes result in the release of cytochrome $c$ and initiate the caspase signaling pathway.

In conclusion, the present study found that ATL may activate cofilin through the targeted inhibition of LIMK enzyme activity. ATL can upregulate the ratio of $\mathrm{G} / \mathrm{F}$-actin, and inhibit the migration and invasion of GBM cells. Activated cofilin and $\mathrm{G}$-actin can be co-transferred to the mitochondria to initiate the mitochondrial/cytochrome $c$ pathway to induce apoptosis (Fig. 8). Combined with previous findings (6), it was confirmed that ATL acts on multiple pathways and has multiple targets through which it exerts its anticancer effects on GBM. The data of the present study highlight the therapeutic potential of ATL as a natural product in the treatment of GBM and support its further development for clinical applications.

\section{Acknowledgements}

Not applicable.

\section{Funding}

The present study was supported by grants from the Liaoning Provincial Natural Science Foundation of China (grant nos. 2019-BS-05, 2019-ZD-1009, 20180550761, 20180550976 and 2017010889-301), the Science and Technology Innovation Fund Project of Dalian (grant no. 2019J13SN105), and the Health Commission Foundation of Dalian (grant nos. $17 Z 1007$ and 1911032).

\section{Availability of data and materials}

All data generated or analyzed during the present study are included in this published article.

\section{Authors' contributions}

LJZhang, XY and XW initiated the study and designed the experiments. XW, SZ, TR and XYL performed the experiments. XW, LJZhao and LFY analyzed the data. XW, SZ, TR and XYL prepared the figures. XW, SZ and TR wrote the manuscript. All authors reviewed the manuscript. All authors read and approved the final manuscript.

\section{Ethics approval and consent to participate}

All procedures were in performed in line with the National Institutes of Health Guidelines for the Care and Use of Laboratory Animals (National Institutes of Health, Bethesda, Maryland, USA). The experimental protocol was approved by the Animal Care and Ethics Committee of Dalian Medical University.

\section{Patient consent for publication}

Not applicable.

\section{Competing interests}

The authors declare that they have no competing interests.

\section{References}

1. Bryukhovetskiy I, Ponomarenko A, Lyakhova I, Zaitsev S, Zayats Y,Korneyko M,Eliseikina M, Mischenko P, Shevchenko V, Shanker Sharma H, et al: Personalized regulation of glioblastoma cancer stem cells based on biomedical technologies: From theory to experiment (Review). Int J Mol Med 42: 691-702, 2018.

2. Jian S, Chen L, Minxue L, Hongmin C, Ronghua T, Xiaoxuan F, Binbin $Z$ and Shiwen G: Tanshinone I induces apoptosis and protective autophagy in human glioblastoma cells via a reactive oxygen species-dependent pathway. Int J Mol Med 45: 983-992, 2020.

3. Hwang TW, Kim DH, Kim DB, Jang TW, Kim GH, Moon M, Yoon KA, Choi DE, Park JH and Kim JJ: Synergistic anticancer effect of acteoside and temozolomide-based glioblastoma chemotherapy. Int J Mol Med 43: 1478-1486, 2019.

4. Butowski NA, Sneed PK and Chang SM: Diagnosis and treatment of recurrent high-grade astrocytoma. J Clin Oncol 24: 1273-1280, 2006.

5. Cantrell CL, Abate L, Fronczek FR, Franzblau SG, Quijano L and Fischer NH: Antimycobacterial eudesmanolides from Inula helenium and Rudbeckia subtomentosa. Planta Med 65: 351-355, 1999.

6. Wang X, Yu Z, Wang C, Cheng W, Tian X, Huo X, Wang Y, Sun C, Feng L, Xing J, et al: Alantolactone, a natural sesquiterpene lactone, has potent antitumor activity against glioblastoma by targeting IKK $\beta$ kinase activity and interrupting NF- $\kappa \mathrm{B} / \mathrm{COX}-2$-mediated signaling cascades. J Exp Clin Cancer Res 36: 93, 2017.

7. Lauffenburger D and Horwitz A: Cell migration: A physically integrated molecular process. Cell 84: 359-369, 1996.

8. Mounier N and Arrigo AP: Actin cytoskeleton and small heat shock proteins: How do they interact? Cell Stress Chaperones 7: $167-176,2002$.

9. Huang T, DerMardirossian C and Bokoch G: Cofilin phosphatases and regulation of actin dynamics. Curr Opin Cell Biol 18: 26-31, 2006.

10. Zhang W, Gan $\mathrm{N}$ and Zhou J: Immunohistochemical investigation of the correlation between LIM kinase 1 expression and development and progression of human ovarian carcinoma. J Int Med Res 40: 1067-1073, 2012.

11. Zhou Y, Su J, Shi L, Liao Q and Su Q: DADS downregulates the Rac1-ROCK1/PAK1-LIMK1-ADF/cofilin signaling pathway, inhibiting cell migration and invasion. Oncol Rep 29: 605-612, 2013. 
12. Li R, Doherty J, Antonipillai J, Chen S, Devlin M, Visser K, Baell J, Street I, Anderson RL and Bernard O: LIM kinase inhibition reduces breast cancer growth and invasiveness but systemic inhibition does not reduce metastasis in mice. Clin Exp Metastasis 30: 483-495, 2013.

13. Aggelou H, Chadla P, Nikou S, Karteri S, Maroulis I, Kalofonos H, Papadaki H, and Bravou V: LIMK/cofilin pathway and slingshot are implicated in human colorectal cancer progression and chemoresistance. Virchows Arch 472: 727-737, 2018.

14. Karbowski $M$ and Youle RJ: Dynamics of mitochondrial morphology in healthy cells and during apoptosis. Cell Death Differ 10: 870-880, 2003

15. Khan M, Yi F, Rasul A, Li T, Wang N, Gao H, Gao R and Ma T: Alantolactone induces apoptosis in glioblastoma cells via GSH depletion, ROS generation, and mitochondrial dysfunction. IUBMB life 64: 783-794, 2012.

16. Chen H, Bernstein B and Bamburg J: Regulating actin-filament dynamics in vivo. Trends Biochem Sci 25: 19-23, 2000.

17. Tsai NM, Chen YL, Lee CC, Lin PC, Cheng YL, Chang WL, Lin SZ and Harn HJ: The natural compound n-butylidenephthalide derived from angelica sinensis inhibits malignant brain tumor growth in vitro and in vivo. J Neurochem 99: 1251-1262, 2006.

18. Li GB, Cheng Q, Liu L, Zhou T, Shan CY, Hu XY, Zhou J, Liu EH, Li P and Gao N: Mitochondrial translocation of cofilin is required for allyl isothiocyanate-mediated cell death via ROCK1/PTEN/PI3K signaling pathway. Cell Commun Signal 11: 50, 2013.

19. Van Troys M, Huyck L, Leyman S, Dhaese S, Vandekerkhove J and Ampe C: Ins and outs of ADF/cofilin activity and regulation. Eur J Cell Biol 87: 649-667, 2008.

20. Bernstein BW and Bamburg JR: ADF/cofilin: A functional node in cell biology. Trends Cell Biol 20: 187-195, 2010.

21. Lei JC, Yu JQ, Yin Y, Liu YW and Zou GL: Alantolactone induces activation of apoptosis in human hepatoma cell. Food Chem Toxicol 50: 3313-3319, 2012.

22. Mi XG, Song ZB, Wu P, Zhang YW, Sun LG, Bao YL, Zhang Y, Zheng LH, Sun Y, Yu CL, et al: Alantolactone induces cell apoptosis partially through down-regulation of testes-specific protease 50 expression. Toxicol Lett 224: 349-355, 2014.

23. Shi Y, Bao YL, Wu Y, Yu CL, Huang YX, Sun Y, Zheng LH and Li YX: Alantolactone inhibits cell proliferation by interrupting the interaction between Cripto-1 and activin receptor type II A in activin signaling pathway. J Biomol Screen 16: 525-535, 2011.

24. Ridley AJ, Schwartz MA, Burridge K, Firtel RA, Ginsberg MH, Borisy G, Parsons JT and Horwitz AR: Cell migration: Integrating signals from front to back. Science 302: 1704-1709, 2003

25. Campellone KG and Welch MD: A nucleator arms race: Cellular control of actin assembly. Nat Rev Mol Cell Biol 11: 237-251, 2010.
26. Hitchcock-Degregori SE: Chemotaxis: Cofilin in the driver's seat. Curr Biol 16: R1030-R1032, 2006.

27. Lee MH, Kundu JK, Chae JI and Shim JH: Targeting ROCK/LIMK/cofilin signaling pathway in cancer. Arch Pharm Res 42: 481-491, 2019.

28. Berger K and Moeller MJ: Cofilin-1 in the podocyte: A molecular switch for actin dynamics. Int Urol Nephrol 43: 273-275, 2011.

29. Wang W, Mouneimne G, Sidani M, Wyckoff J, Chen X, Makris A, Goswami S, Bresnick AR and Condeelis JS: The activity status of cofilin is directly related to invasion, intravasation, and metastasis of mammary tumors. J Cell Biol 173: 395-404, 2006.

30. Pandey D, Goyal P and Siess W: Lysophosphatidic acid stimulation of platelets rapidly induces $\mathrm{Ca}^{2+}$-dependent dephosphorylation of cofilin that is independent of dense granule secretion and aggregation. Blood Cells Mol Dis 38: 269-279, 2007.

31. Yang C, Yang J, Sun M, Yan J, Meng X and Ma T: Alantolactone inhibits growth of K562/adriamycin cells by downregulating $\mathrm{Bcr} / \mathrm{Abl}$ and P-glycoprotein expression. IUBMB Life 65: 435-444, 2013.

32. Lei JC, Yu JQ, Yin Y, Liu YW and Zou GL: Alantolactone induces activation of apoptosis in human hepatoma cells. Food Chem Toxicol 50: 3313-3319, 2012.

33. Ding Y, Wang H, Niu J, Luo M, Gou Y, Miao L, Zou Z and Cheng Y: Induction of ROS overload by alantolactone prompts oxidative DNA damage and apoptosis in colorectal cancer cells. Int J Mol Sci 17: 558, 2016.

34. Pal HC, Sehar I, Bhushan S, Gupta BD and Saxena AK: Activation of caspases and poly (ADP-ribose) polymerase cleavage to induce apoptosis in leukemia HL-60 cells by Inula racemosa. Toxicol In Vitro 24: 1599-1609, 2010

35. Dalla Via L, García-Argáez A, Martínez-Vázquez M, Grancara S, Martinis P and Toninello A: Mitochondrial permeability transition as target of anticancer drugs. Curr Pharm Des 20: 223-244, 2014.

36. Kanellos $\mathrm{G}$ and Frame MC: Cellular functions of the ADF/cofilin family at a glance. J Cell Sci 129: 3211-3218, 2016.

37. Chua BT, Volbracht C, Tan KO, Li R, Yu VC and Li P: Mitochondrial translocation of cofilin is an early step in apoptosis induction. Nat Cell Biol 5: 1083-1089, 2003.

38. Rehklau K, Gurniak CB, Conrad M, Friauf E, Ott M and Rust MB: ADF/cofilin proteins translocate to mitochondria during apoptosis but are not generally required for cell death signaling. Cell Death Differ 19: 958-967, 2012.

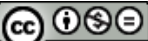

This work is licensed under a Creative Commons

Attribution-NonCommercial-NoDerivatives 4.0 International (CC BY-NC-ND 4.0) License. 